\title{
A Systematic Review and Meta-Analysis of the Effect of Lifestyle Modification on Metabolic Control in Overweight Children
}

\author{
Angela Shin-Yu Lien, ${ }^{1,2}$ Jia-Ling Tsai, ${ }^{1,3}$ Jian-Tao Lee, ${ }^{1}$ Mei-Yao Wu, ${ }^{4}$ \\ Yi-Der Jiang, ${ }^{5}$ and Hung-Rong Yen ${ }^{4,6,7,8,9}$ \\ ${ }^{1}$ School of Nursing, College of Medicine, Chang Gung University, Taoyuan, Taiwan \\ ${ }^{2}$ Division of Endocrinology and Metabolism, Department of Internal Medicine, Chang Gung Memorial Hospital, \\ Linkou Branch, Taoyuan, Taiwan \\ ${ }^{3}$ School of Nursing, College of Medicine, National Taiwan University, Taipei, Taiwan \\ ${ }^{4}$ Research Center for Traditional Chinese Medicine, Department of Medical Research, China Medical University Hospital, \\ Taichung, Taiwan \\ ${ }^{5}$ Department of Internal Medicine, National Taiwan University Hospital, Taipei, Taiwan \\ ${ }^{6}$ Department of Chinese Medicine, China Medical University Hospital, Taichung, Taiwan \\ ${ }^{7}$ Research Center for Chinese Herbal Medicine, China Medical University, Taichung, Taiwan \\ ${ }^{8}$ School of Chinese Medicine, China Medical University, Taichung, Taiwan \\ ${ }^{9}$ Department of Biotechnology, Asia University, Taichung, Taiwan
}

Correspondence should be addressed to Yi-Der Jiang; yiderj@ntu.edu.tw and Hung-Rong Yen; hungrongyen@gmail.com

Received 20 February 2017; Accepted 21 June 2017; Published 27 September 2017

Academic Editor: Kieran Cooley

Copyright ( 2017 Angela Shin-Yu Lien et al. This is an open access article distributed under the Creative Commons Attribution License, which permits unrestricted use, distribution, and reproduction in any medium, provided the original work is properly cited.

Childhood obesity is associated with type 2 diabetes mellitus. We aimed to determine the effects of lifestyle modification programs on fasting plasma glucose (FPG) levels in overweight children. We queried six relevant electronic databases and manually searched for studies published before December 2016. Overweight/obese children who underwent a lifestyle modification for more than 6 months were included. A total of 3923 children from eight randomized controlled trials (RCTs) were included. Compared with the control group, the lifestyle modification group had significantly lower FPG levels by $1.3 \mathrm{mg} / \mathrm{dL}$. The mean differences were significantly decreased for both secondary outcomes; BMI $z$-score decreased by 0.16 units and insulin levels decreased by $2.4 \mathrm{mU} / \mathrm{L}$. The metaregression showed that the follow-up duration was associated with FPG levels and BMI and insulin levels and half year is a suitable follow-up duration for this population. This study showed that lifestyle modification programs may be effective in reducing the FPG levels of overweight/obese children. Further high-quality RCTs with longer follow-up periods are needed to evaluate the long-term effect of this complementary approach for diabetes mellitus prevention on overweight/obese children.

\section{Introduction}

Childhood obesity is one of the most important health challenges that pose long-term impacts on medical, psychosocial, and financial consequences [1,2]. In 2010, the estimated number of overweight children under 5 years old was more than 42 million worldwide. Nearly 35 million of these children lived in developing countries, mostly in urban settings [3]. The prevalence of overweight children between 5 and 12 years old in the United States, Italy, Greece, and Spain is greater than 30\% [4]. An increased prevalence of overweight children has also been identified in Taiwan. The prevalence of overweight Taiwanese children aged 6 to 12 years was $29.8 \%$ for boys and $25.1 \%$ for girls [5].

Type 2 diabetes was known as an "adult-onset" disease. However, the increasing prevalence of type 2 diabetes in children parallels the increasing incidence of obesity worldwide. Overweight and obese youth are also associated with cardiovascular disease and type 2 diabetes due to the accumulation of body fat, which is highly correlated with fasting insulin 
release [6]. In 2000, nearly $45 \%$ of new pediatric cases with type 2 diabetes were overweight, sharply increasing from $4 \%$ in the 1990s [2]. According to the HEALTHY study that involved 6,358 sixth-grade pupils, $49.3 \%$ of children had a body mass index (BMI) in the 85th percentile or higher, and $16.0 \%$ demonstrated fasting plasma glucose (FPG) levels greater than $100 \mathrm{mg} / \mathrm{dL}$ even though they did not have signs and symptoms of diabetes [7]. Type 2 diabetes is highly associated with obesity and plasma glucose level. Race (particularly minority populations), obesity, and family history were prominent risk factors related to adolescent type 2 diabetes [8]. An increased waist circumference was a strong risk factor for type 2 diabetes as well [6]. In a nationwide urine-screening program for nearly 3 million school children in Taiwan, the odds of type 2 diabetes mellitus were 18.8 times higher for those with a BMI over the 95th percentile relative to those with a BMI below the 50th percentile [9]. Mortality, atherosclerotic cardiovascular disease, and complication rates were higher for patients with young type 2 than for those with type 1 diabetes [10].

Strategies to control body weight and to address the related complications of obesity are important issues for pediatric healthcare. In an ecological context, unhealthy behavior, lifestyle, and environmental issues all contribute to childhood obesity. Therefore, lifestyle modification has been applied as complementary therapy to prevent children from obesity, diabetes, and cardiovascular diseases. Increased sedentary behavior, an energy imbalance, an excessive caloric intake, and poor dietary components are major elements of lifestyle that contribute to obesity [11]. Pharmaceutical and surgical therapies may be helpful to quickly reduce extreme body weight, but lifestyle interventions that encourage an increase in physical activity and the consumption of healthy foods are essential to maintain a long-term behavior change for better type 2 diabetes management [12]. In the United Kingdom Prospective Diabetes Study (UKPDS), diabetic patients improved glycemic control and decreased other cardiovascular risk factors through standard lifestyle regulation or adjunctive medical or surgical interventions for severe hyperglycemia [13]. Overweight/obesity not only contributes to poor health but also affects the psychological and social well-being of children. Furthermore, it affects the economy. Over the last 20 years, annual obesity-related hospital costs have increased 3-fold, reaching \$127 million per year in the United States [10]. However, despite its impact on health and psychological and social well-being and economic impact, obesity can be prevented through early lifestyle modifications in childhood [14].

In the Western countries, "complementary and alternative" usually refer to the context in which a therapy is used. So a therapy can be complementary if it is used alongside conventional treatments or alternative if it is used in place of a conventional treatment. Lifestyle modifications, including a healthy diet, weight control, increased physical activity, and reduced sedentary behavior, have been recommended as a complementary therapy for the management of obese and hyperglycemic subjects to prevent or delay the onset of type 2 diabetes [15] and are consistent with evidence gathered from adult populations [16-21]. The American Diabetes
Association recently revised its strategic plan for lifestyle modification; it called for increasing focus on the translation of prevention research into strategies for primary care and community treatment to reduce the risk of developing type 2 diabetes [22, 23].

Many clinical trials have been conducted in schools, hospitals, families, and communities but have led to mixed results $[24,25]$. Some trials effectively improved knowledge or skills, but most failed to prevent obesity or decrease blood glucose, insulin, and other risk factors in children. A randomized, controlled pilot study indicated that Exenatide ${ }^{\circledR}$ can significantly reduce body mass index for extreme obesity, but it cannot reduce FPG [23]. Moderate to vigorous physical activity can lower waist circumference, blood pressure, insulin level, and triglycerides in children and adolescents [26]. However, the effects of lifestyle modifications, the most important nonpharmaceutical approach, on glucose control or diabetes prevention have not been well established. In addition, studies on youth have not utilized impaired glucose tolerance (IGT) as a marker of progression toward type 2 diabetes, and the long-term effectiveness of obesity treatment and prevention programs provided for children need to be clarified. To fill the gap, we conducted a systematic review that searched for lifestyle modifications as the intervention in clinical trials. Then, we applied meta-analytical methods to determine the effect of lifestyle modifications on FPG as the primary outcome and BMI or BMI $z$-score, fasting insulin level, and $\mathrm{HbA}_{1 \mathrm{C}}$ as the secondary indicators among overweight/obese children to make a conclusion.

\section{Material and Methods}

2.1. Definition of Overweight and Obesity in Children. The World Health Organization (WHO) has suggested the use of BMI as an indicator of extreme body weight. BMI is a simple index of the weight-to-height ratio $\left(\mathrm{kg} / \mathrm{m}^{2}\right)$ that been used commonly to classify individuals as underweight, overweight, or obese. This study adopted the definition of the International Obesity Task Force (IOTF) and the references of the WHO to classify individuals as underweight, overweight, or obese $[28,29]$. An overweight child has a BMI that falls between the 85th and 95th percentile after adjustment for gender and age; this is approximately equivalent to the BMI range from 25 to $29.99 \mathrm{~kg} / \mathrm{m}^{2}$ in adults (the normal range for adults is 18.5 to $24.99 \mathrm{~kg} / \mathrm{m}^{2}$; for Asian adults, the cut-off point is 23). Likewise, an obese youth has a BMI that lies above the 95th percentile, equivalent to at least $30 \mathrm{~kg} / \mathrm{m}^{2}$ in adults (for Asian adults, the cut-off point is 27 , and it considers age and gender) [30].

2.2. Study Strategy and Eligibility Criteria. This review integrated the results from relevant studies by following the systematic review and meta-analysis guidelines outlined in the Preferred Reporting Items for Systematic Review and Meta-Analysis (PRISMA) statement [31]. The authors conducted a comprehensive literature search of the PubMed, Cochrane, EMBASE, Cinahl, ProQuest dissertation and thesis, and PsychINFO databases and manually searched for studies published before December 2016. There were 
no limitations based on language, and the search focused on the influence of lifestyle interventions (more than 6 months) on FPG levels in overweight/obese children. The search strategy included the following keywords and $\mathrm{MeSH}$ terms: "randomized control trial" OR "clinical control trial" AND "childhood overweight and obesity" AND "lifestyle," AND "diabetes" AND "exercise" OR "physical activity” OR "fitness." Studies that evaluated subjects with certain diseases or conditions (e.g., neurosurgical, cardiovascular, diabetes mellitus, eating disorders, malnutrition, and psychosocial or physical disabilities) were excluded, along with those that involved the participation of subjects in interventions other than lifestyle modification (e.g., breastfeeding) or that provided pharmacological treatment for obesity.

Using this method, we identified 572 articles, including 5 relevant systematic reviews retrieved by manual search. After 332 duplicates studies were removed, the abstracts of all of the identified articles were reviewed independently by 2 authors. Overweight children under the age of 18 were included in the review. The intervention was limited to lifestyle modification programs that included diet control, healthy nutrition, exercise, fitness, and/or physical activities. The primary outcome was FPG levels, and the secondary outcome indicators were BMI or BMI $z$-scores, fasting insulin level, and $\mathrm{HbA}_{1 \mathrm{C}}$. Furthermore, an article was excluded if the full text was not available or if it was not an original study.

After these criteria were applied, 127 articles with full text remained, of which we excluded 70 studies that did not report FPG, 26 studies that included lifestyle programs which only provided an exercise intervention, 9 studies that were not peer-reviewed original studies, 10 studies that had a followup period less than 6 months or that fulfilled other exclusion criteria, and 3 studies that mixed both normal weight and overweight children [32-34]. The remaining 9 studies were included in the qualitative synthesis and meta-analysis [3543]. The full texts of the included articles were reviewed independently by 2 authors, and disagreements were resolved by consensus. A detailed screening diagram is shown in Figure 1.

2.3. Data Extraction. The 9 studies included in the metaanalysis were abstracted systematically for details and characteristics, including study location, design, subjects, intervention style (i.e., lifestyle modification program), and study period (Table 1). The primary outcome of FPG and the secondary outcomes of BMI or BMI $z$-score, insulin level, and $\mathrm{HbA}_{1 \mathrm{C}}$ were taken from the references of the included articles (Tables 2 and 3). During the systematic review, 2 reviewers extracted data independently using a standardized data extraction form. To resolve assessment differences, the reviewers deliberated until a consensus was reached.

2.4. Quality Assessment. We carefully reviewed the design, conduct, analysis, and reporting of each study and considered whether there was any risk of bias that would make the 2 groups noncomparable. We used a domain-based approach with Cochrane risk of bias tool [44] to assess the quality of each included study. It contains the following domains: (1) random sequence generation, (2) allocation concealment, (3) blinding of participants, (4) personnel blinding of outcome assessment, (5) incomplete outcome data, (6) selective outcome reporting, and (7) other sources of bias (see Figure S1 in Supplementary Material available online at https://doi.org/10.1155/2017/5681909). The judgment of whether there was high, low, or unknown bias was reached by consensus between 2 reviewers.

2.5. Analysis and Synthesis Methods. Qualitative synthesis and the random-effects model meta-analysis were performed using Comprehensive Meta-Analysis (CMA) software Version 2.2; it combined studies under the random-effects model and compared the mean changes in FPG levels between the intervention and control groups [45]. We calculated difference in means and 95\% confidence intervals (CI) for estimate the effect between lifestyle intervention and control for overweight/obese children.

\section{Results}

3.1. Study Population Characteristics. In total, there were 3923 subjects, of whom 1943 subjects were in the intervention group and 1980 in the control group. Five studies were performed in the United States with subjects aged 9 14 years (one for Mexican-Americans and one for Latinos), two were in the Netherlands with subjects aged $8 \sim 17$, and the remained 2 studies were one from West Germany [40] and one was from China [43]. Six studies recruited 41 81 children in a single center with a treatment lasting within $6 \sim 12$ months, one study collects data from school cluster sampling [43], and only one multiple center study was performed as part of the HEALTHY study [37], which was a much larger study that recruited more than a thousand overweight kids in each group with a treatment lasting within 1 3 years (Table 1). But none of any included studies report the issue of gender difference.

3.2. Outcome Indicators. For fasting glucose levels, for the primary outcome, the results are summarized in Table 2. One study showed no change in either the intervention or control group. Five studies showed greater FPG reduction in the intervention group than in the control group; however, none of the differences were statistically significant. Three articles showed a decrease in FPG for the intervention group and an increase for the control group. Two articles reported a decrease in FPG for both groups, and three studies reported an increase in FPG for both groups. Regarding BMI $z$-scores, four studies showed a decrease for both the intervention and control groups, two articles showed a decrease for the intervention group and an increase for the control group, one article showed no change for the intervention group but a decrease for the control group, and the remaining study showed an increase for the intervention group and no change in the control group. Regarding insulin level changes, four articles reported an increase for both the intervention and control groups, two articles reported a decrease for both groups, one article reported a decrease for the intervention group but an increase for the control group, the last one report no data. Among the 9 studies, only three of them reported $\mathrm{HbA}_{1 \mathrm{C}}$ level (Table 3). 


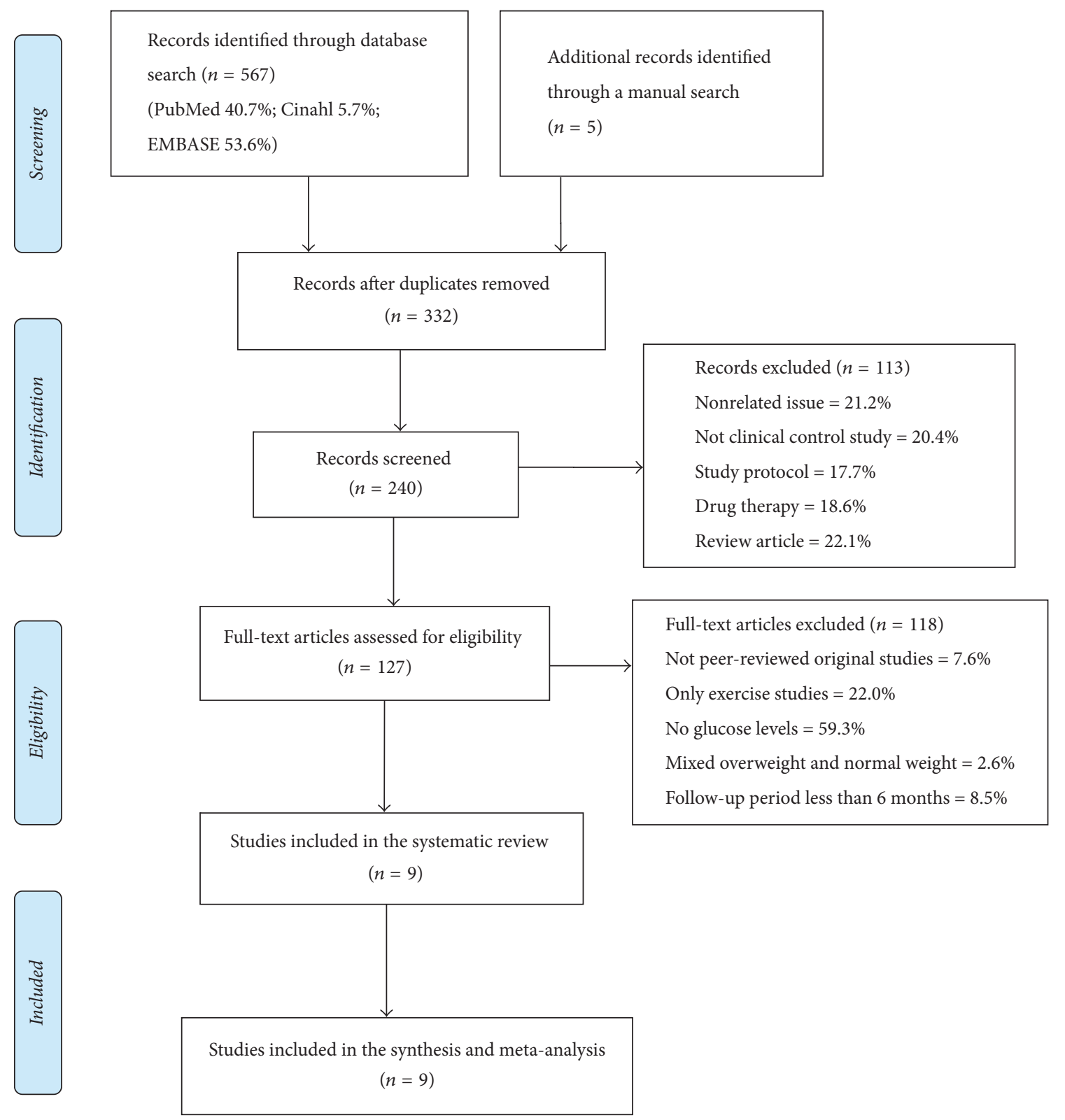

FIGURE 1: The study selection and screening diagram. From [27].

Regarding other biomarkers, two articles showed changes in the insulin resistance index (homeostasis model assessment, HOMA) for both groups. Seven studies reported changes in the lipid profile (triglyceride, high density lipoprotein, and low density lipoprotein), of which one showed an inverse effect on both groups and two found no effect on either group.

3.3. Meta-Analysis. We pooled all of the studies into a metaanalysis, and the forest plot of the random-effects model showed moderate heterogeneity, as demonstrated by the high $I^{2}$ value $(86.49 \%)$. The point estimate of the mean difference was $-1.282 \mathrm{mg} / \mathrm{dL}$ (95\% CI, -2.14 to $-0.42, Z=-2.92, p=$ 0.003 ), which indicated that lifestyle modifications decreased the mean fasting glucose levels by $1.3 \mathrm{mg} / \mathrm{dL}$. The sensitivity test indicated that the omission of any study would change the estimate between -0.66 ( -11.43 to 5.60$) \mathrm{mg} / \mathrm{dL}$ and -4.71 $(-21.93$ to -9.04$) \mathrm{mg} / \mathrm{dL}$. In addition, we analyzed the change in the secondary outcome variables of BMI $z$-score and insulin level among the seven studies. After the data were pooled for the meta-analysis, the effect sizes of the mean differences in BMI $z$-scores and insulin levels were 0.06 units $(95 \% \mathrm{CI}=-0.27$ to $-0.05, Z=-2.90, p=0.004)$ and $-2.4 \mathrm{mU} / \mathrm{L}$ ( $95 \% \mathrm{CI}=-3.91$ to $-0.89, Z=-3.12, p=0.002$ ). The results indicated a significant decrease in the secondary outcome variables due to lifestyle interventions between the 2 groups from baseline to the end of the study. We also found that the change of $\mathrm{HbA}_{1 \mathrm{C}}$ level was $-0.08 \%$. However, there was no significant difference $(95 \% \mathrm{CI}=-0.17$ to $0.21, Z=$ $-1.54, p=0.12$ ) (Figure 2). 


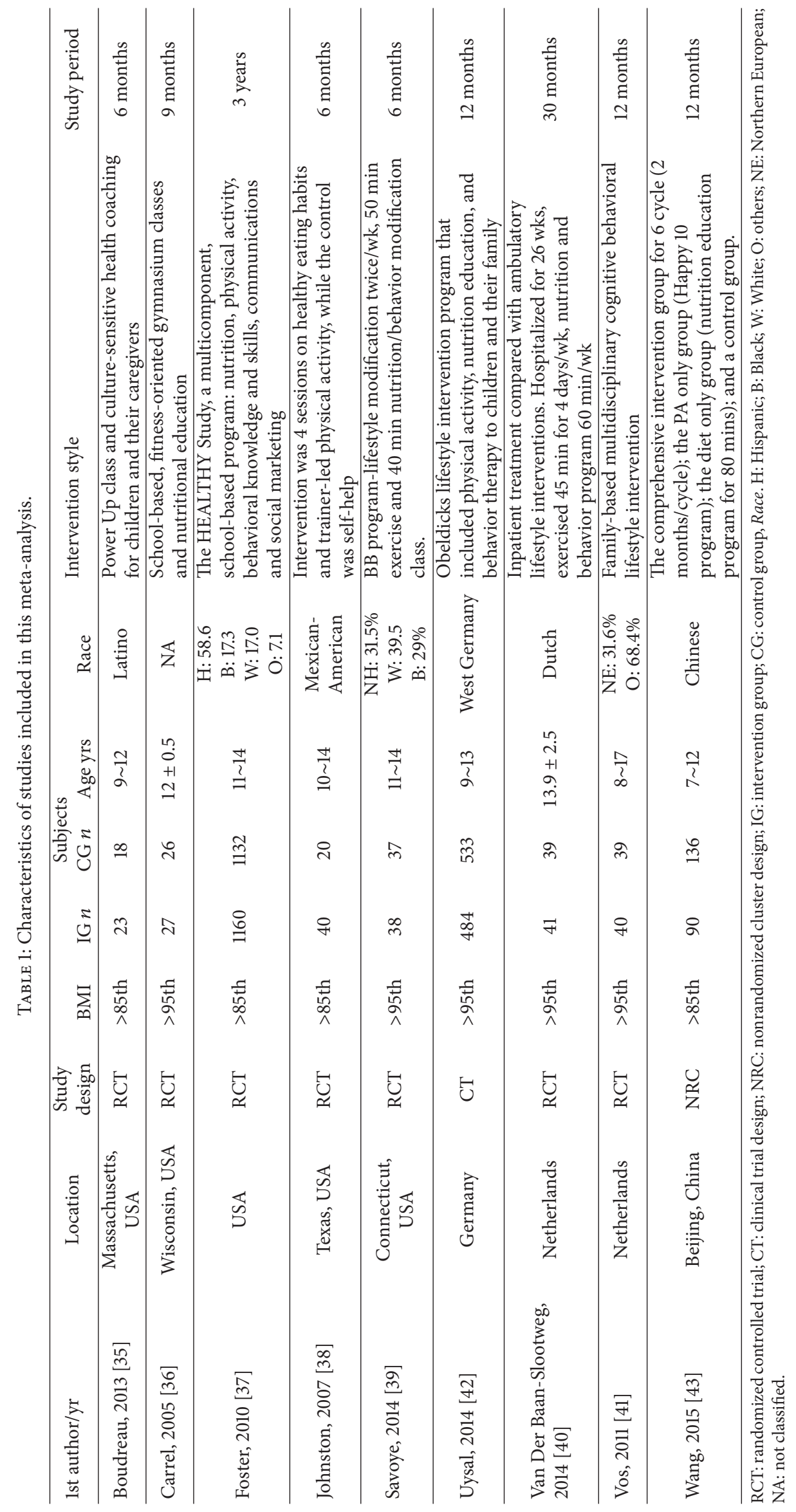




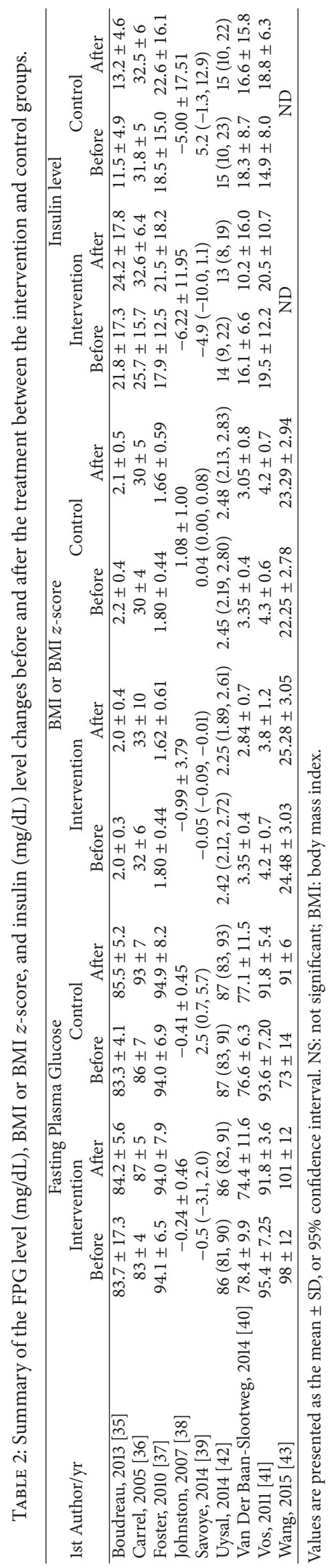


TABLE 3: Summary of the $\mathrm{HbA}_{1 \mathrm{C}}$ [\%] changes before and after the treatment between the intervention and control groups.

\begin{tabular}{|c|c|c|c|c|}
\hline \multirow{3}{*}{ 1st author/yr } & \multicolumn{4}{|c|}{$\mathrm{HbA}_{1 \mathrm{C}}[\%]$} \\
\hline & \multicolumn{2}{|c|}{ Intervention } & \multicolumn{2}{|c|}{ Control } \\
\hline & Before & After & Before & After \\
\hline Boudreau, 2013 [35] & $5.6 \pm 0.3$ & $5.7 \pm 0.3$ & $5.6 \pm 0.3$ & $5.7 \pm 0.3$ \\
\hline Savoye, 2014 [39] & \multicolumn{2}{|c|}{$-0.16(-0.27,-0.05)$} & \multicolumn{2}{|c|}{$-0.05(-0.17,0.07)$} \\
\hline Uysal, 2014 [42] & $5.4(5.2,5.7)$ & $5.5(5.3,5.7)$ & $5.5(5.3,5.7)$ & $5.5(5.3,5.8)$ \\
\hline
\end{tabular}

The results from both asymmetry funnel plot and Egger's linear regression test $(t=2.55, p=0.004)$ showed significant publication bias of all eligible studies (Figure S2).

The high $I^{2}$ values for the FPG (86.49\%, Egger's value, $t=$ $2.55, p=0.04$ ), BMI $z$-score (83.05\% Egger's value, $t=2.29$, $p=0.07)$, and insulin level (75.45\%, Egger's value, $t=$ $2.69, p=0.04$ ) analyses indicated heterogeneity (Figure S2), and the meta-regression showed an association of these variables with follow-up length (Figure S3). A further subgroup analysis was performed dividing the studies into 2 groups according to follow-up periods: group 1 for 6 months and group 2 between 9 months and 3 years. The results are shown in Figure 3. Lifestyle interventions significantly reduced FPG by $1.31 \mathrm{mg} / \mathrm{dL}$ ( $95 \% \mathrm{CI},-2.15$ to $-0.47, Z=-3.06, p=0.002$ ), BMI $z$-scores by -0.16 ( $95 \% \mathrm{CI},-0.29$ to $-2.42, Z=-0.03$, $p=0.016)$, and insulin levels by $-2.16 \mathrm{mU} / \mathrm{L}(95 \% \mathrm{CI},-3.62$ to $-0.71, Z=-2.92, p=0.004)$ in the subgroup analysis of the random-effects model (Figure 3 ).

\section{Discussion}

The present meta-analysis reported that patients who received lifestyle modification showed significantly reduced FPG levels by $1.3 \mathrm{mg} / \mathrm{dL}$ compared with patients in the control group. This effect indicated that lifestyle modification interventions could decrease FPG levels, BMI, and insulin levels in overweight/obese children. In adults with IGT or impaired fasting glucose (IFG), lifestyle modification has consistently shown positive results. Previous studies have reported that lifestyle modification programs significantly reduced BMI, body fat, waist circumference, blood sugar levels, glycated hemoglobin $\left(\mathrm{HbA}_{1 \mathrm{C}}\right)$ levels, LDL levels, and HDL levels and prevented the development of type 2 diabetes in high-risk adult subjects $[16,20,21]$. These results supported our findings in a pediatric population. The effect of lifestyle modification shown in this study could act as a pilot and serve as a reference for future high-quality randomized controlled trials.

The Institute of Medicine recommended "an ecological model" for public health interventions, in which the individual subject is viewed "within a larger context of family, community, and society" [46]. During the developmental stages, children require a high amount of energy to grow, making these stages more important than other stages. The American Academy of Pediatrics recently announced the "management of newly diagnosed type 2 diabetes in children and adolescents" [47]. Similar to the management of type 2 diabetes in adults [17], lifestyle modification and Metformin ${ }^{\circledR}$ were recommended as a first-choice therapy, except during hyperglycemic emergencies [47]. This aligns with our study findings that lifestyle modification interventions should be implemented before the onset of the disease. A recent randomized trial tested the dose-dependent benefits of exercise on insulin resistance and body fat in 222 overweight or obese children [48]. A meta-analysis pooled 49 studies on adults to form a conclusion on lifestyle modification programs, which included changing eating habits, consuming healthy food, and increasing fitness and physical activity; they found a reduction in BMI and FPG levels of adults [21]. This was similar to the findings of our study. We applied a subgroup analysis to overcome the high heterogeneity of BMI $z$-scores and insulin levels but not glucose levels. The intervention follow-up length was associated with the outcomes. Specifically, the metaregression revealed that lifestyle interventions were shown to improve FPG levels and BMI $z$-score but not insulin levels or $\mathrm{HbA}_{1 \mathrm{C}}$ changes. Perhaps the small sample size of such studies on children induced potential publication bias. Regardless of exercise intensity, we observed significant reductions in the risk of prediabetes compared with the control group.

The diagnostic criteria for diabetes mellitus in children are the same as those in adults [49]. FPG and $\mathrm{HbA}_{1 \mathrm{C}}$ have been both used as diagnostic criteria for diabetes mellitus in children [9]. However, the oral glucose tolerance test was difficult to perform in children. Other metabolic markers were also difficult to assess because not all of the studies evaluated these markers. This is one of the limitations of this study. This study was also limited by the lack of a standardized physical activity dosage, which made it difficult to compare each study and their reported effectiveness of lifestyle modification, possibly inducing higher heterogeneity. Unlike studies of adult populations, studies of pediatric populations additionally need to develop playful and ageappropriate intervention designs.

In adults with IGT or IFG, lifestyle modification programs have been consistently shown to be effective in the prevention of type 2 diabetes [17-20]. Our study demonstrated that lifestyle modification significantly reduced FPG levels in overweight/obese children. It should be considered that vacations might affect lifestyle regulation as the followup duration was often limited by the semester and school year changes. FPG levels could also be affected by seasonal changes or holidays [50, 51]. Additionally, during puberty, sexual hormones play a more significant role in changing FPG levels $[9,52,53]$. The results may be counterintuitive as the reduction in FPG levels, BMI $z$-score, and insulin levels did not provide a clinically significant meaning. Lifestyle modification interventions are still useful for teenagers. Encouraging exercise in this population is suggested; however, diet cannot be too strict for growing adolescents. 


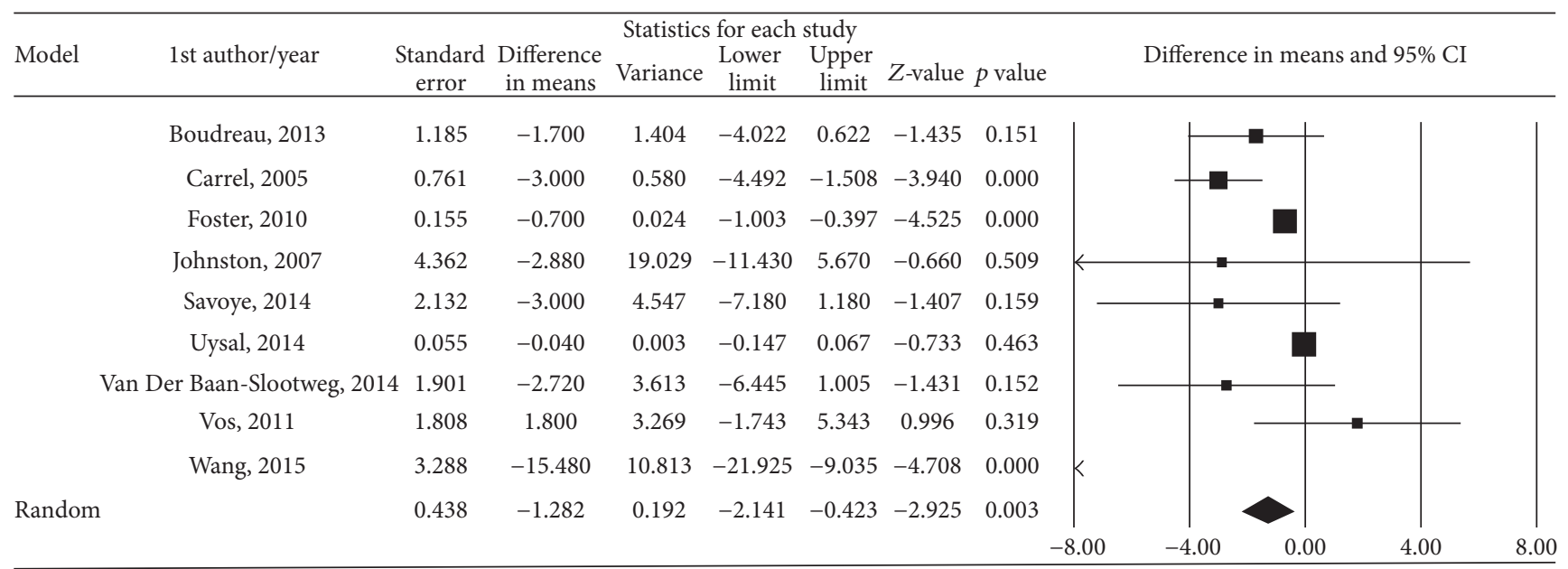

(a) Fasting plasma glucose. Heterogeneity. $Q$-value $=59.25, I^{2}=86.49, p$ value $=0.000$

\begin{tabular}{|c|c|c|c|c|c|c|c|c|c|c|c|c|c|c|}
\hline & & & & & Statistics & for each & study & & & & & & & \\
\hline Model & 1st author/year & Comparison & $\begin{array}{l}\text { Difference } \\
\text { in means }\end{array}$ & $\begin{array}{l}\text { Standard } \\
\text { error }\end{array}$ & Variance & $\begin{array}{c}\text { Lower } \\
\text { limit }\end{array}$ & $\begin{array}{c}\text { Upper } \\
\text { limit }\end{array}$ & $Z$-value & $p$ value & & Differen & means & $5 \% \mathrm{CI}$ & \\
\hline & Boudreau, 2013 & 1 & -0.020 & 0.273 & 0.074 & -0.554 & 0.514 & -0.073 & 0.941 & & & 1 & $\mid$ & \\
\hline & Foster, 2010 & 2 & -0.040 & 0.020 & 0.000 & -0.079 & -0.001 & -1.986 & 0.047 & & & & & \\
\hline & Johnston, 2007 & 1 & -2.070 & 0.421 & 0.177 & -2.895 & -1.245 & -4.917 & 0.000 & & d & & & \\
\hline & Savoye, 2014 & 1 & -0.090 & 0.025 & 0.001 & -0.139 & -0.041 & -3.587 & 0.000 & & & & & \\
\hline & Uysal, 2014 & 2 & -0.200 & 0.051 & 0.003 & -0.299 & -0.101 & -3.945 & 0.000 & & & & & \\
\hline & Van Der Baan-Slootweg, 2014 & 2 & -0.170 & 0.173 & 0.030 & -0.510 & 0.170 & -0.981 & 0.327 & & & & & \\
\hline & Vos, 2011 & 2 & -0.300 & 0.126 & 0.016 & -0.547 & -0.053 & -2.382 & 0.017 & & & & & \\
\hline Random & & & -0.162 & 0.056 & 0.003 & -0.271 & -0.052 & -2.903 & 0.004 & & & $\Delta$ & & \\
\hline & & & & & & & & & & -4.00 & -2.00 & 0.00 & 2.00 & 4.00 \\
\hline
\end{tabular}

(b) BMI $z$-score. Heterogeneity. $Q$-value $=35.40, I^{2}=83.05 \%, p$ value $=0.000$

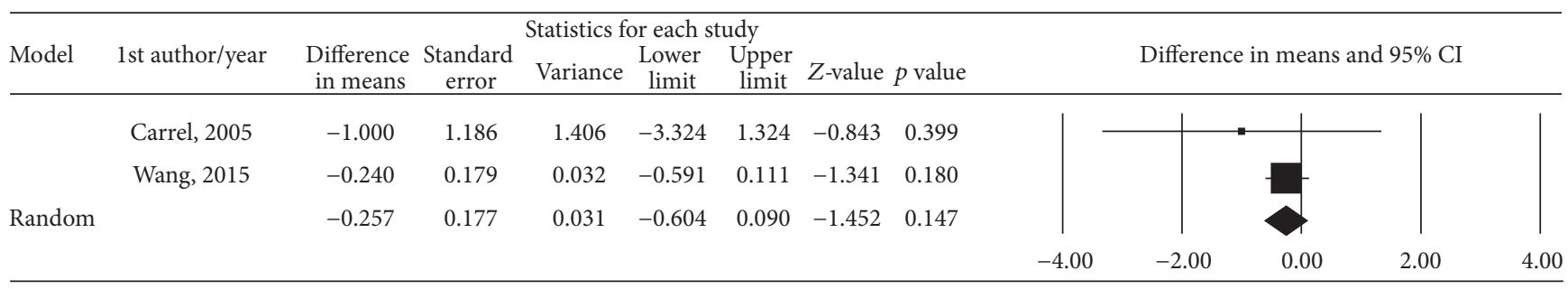

(c) BMI. Heterogeneity. Q-value $=0.402, I^{2}=0 \%, p$ value $=0.526$

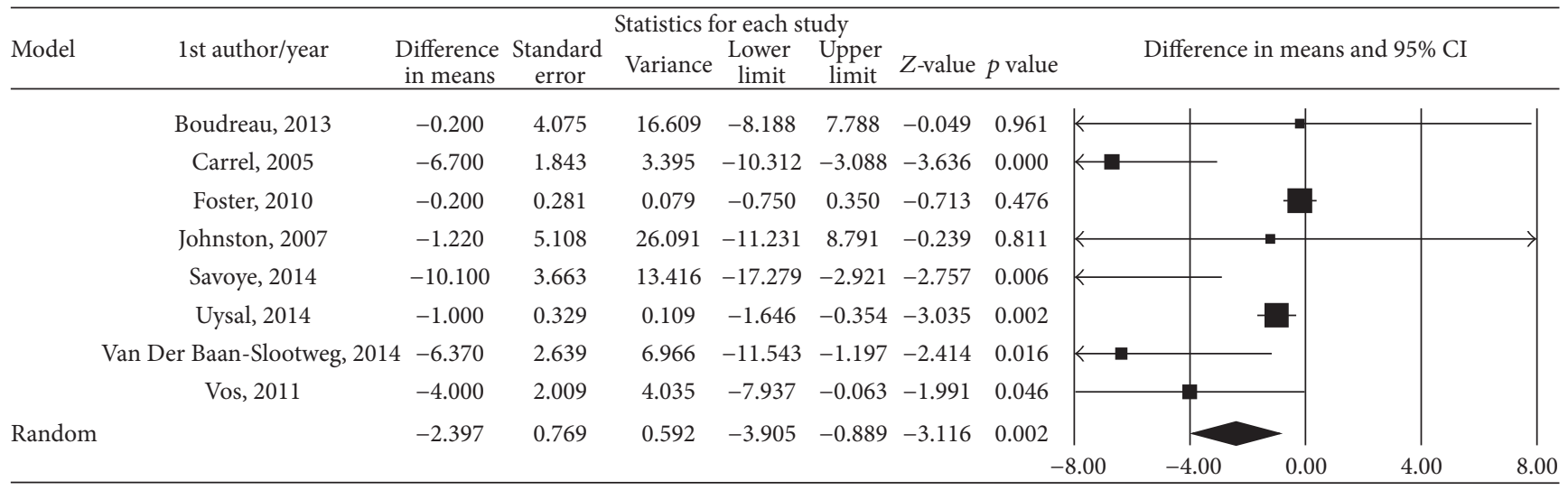

(d) Fasting insulin level. Heterogeneity. $Q$-value $=28.52, I^{2}=75.45 \%$, $p$ value $=0.000$

Figure 2: Continued. 


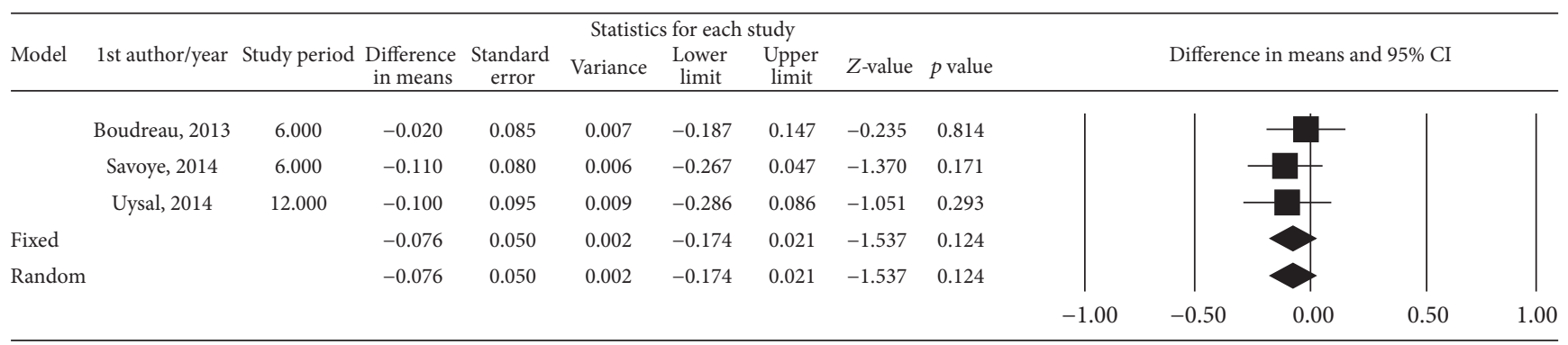

(e) $\mathrm{HbA}_{1 \mathrm{C}}$. Heterogeneity. $\mathrm{Q}$-value $=0.677, \mathrm{I}^{2}=0.00 \%, p$ value $=0.713$

Figure 2: Meta-analysis of the effect size by difference in means.

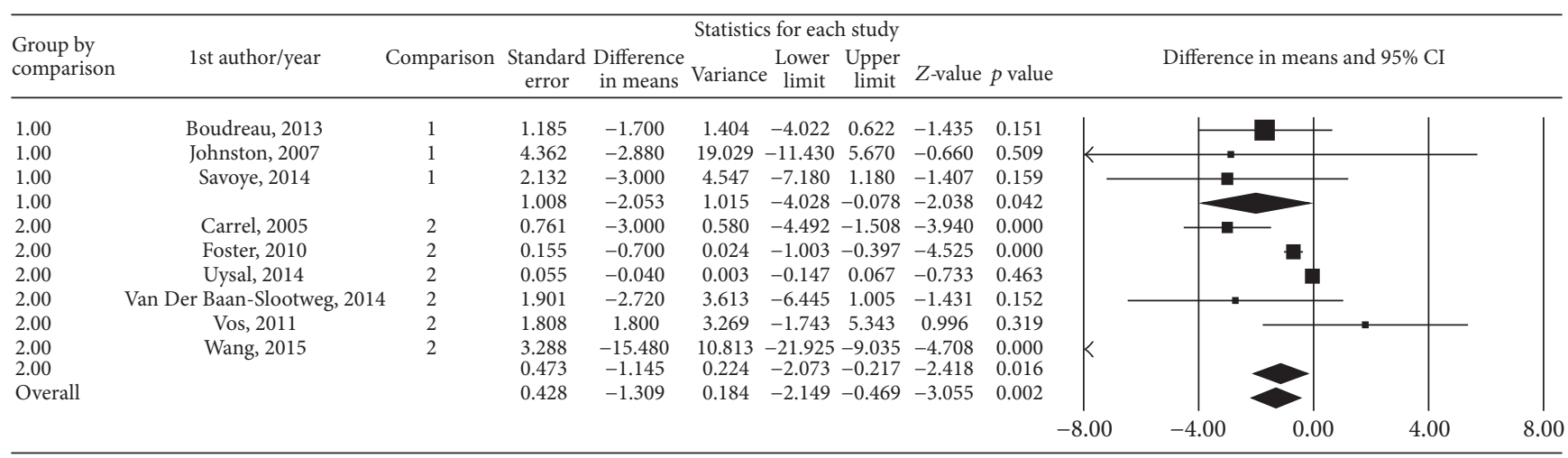

(a) FPG subgroup ( 1 = follow-up length less than $6 \mathrm{M} ; 2$ = follow-up length over $6 \mathrm{M}$ )

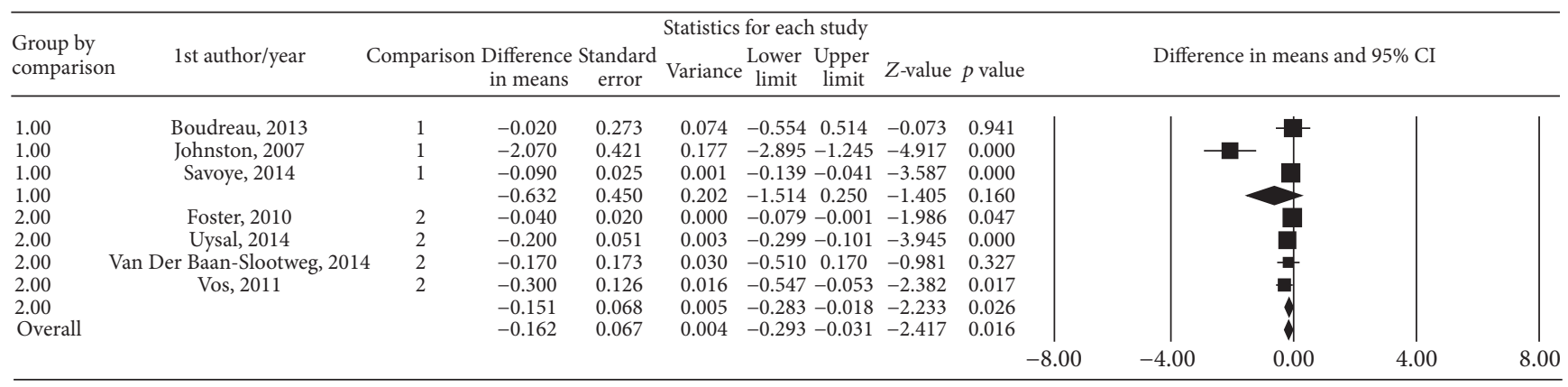

(b) BMI $z$-score $(1=$ follow-up length less than $6 \mathrm{M} ; 2$ = follow-up length over $6 \mathrm{M})$

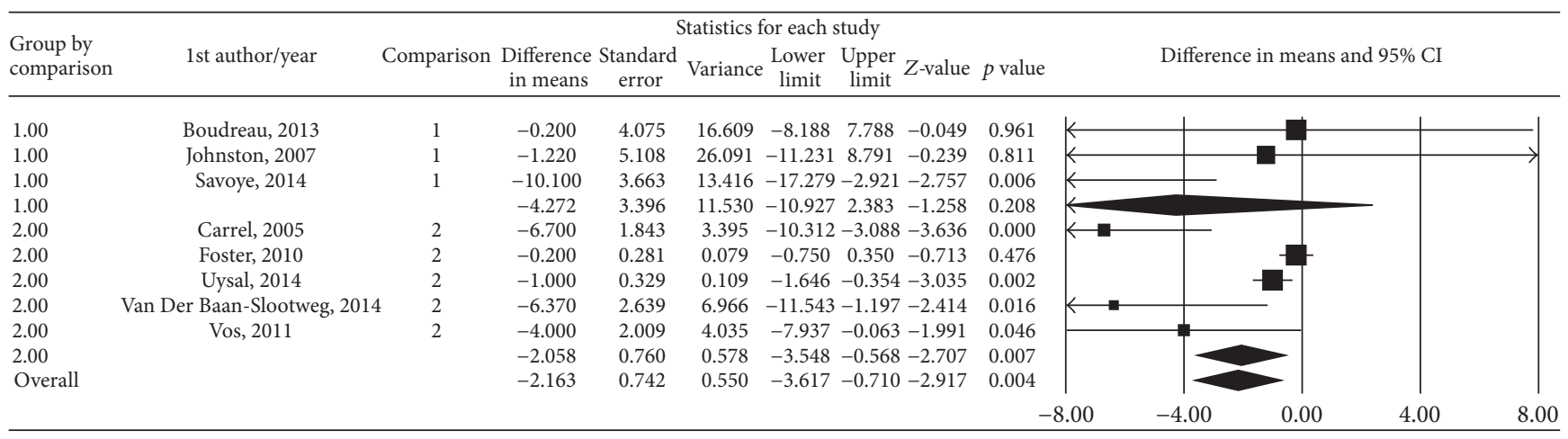

(c) Fasting insulin level ( 1 = follow-up length less than $6 \mathrm{M}$; 2 = follow-up length over $6 \mathrm{M}$ )

Figure 3: Meta-analysis forest plot of the mean changes according to follow-up length: group 1, indicating less than 6 months; group 2 , indicating longer than 6 months. 
It must be noted that 8 of the studies in this review were conducted in high-income countries and that the sample population primarily consisted of Caucasian subjects. Only one study consisted of Chinese population, and this may have acted as a confounding factor as the prevalence of diabetes is dependent on ethnicity. It has to be noted that Asians are more vulnerable to diabetes, despite a lower body mass index on average [54-56]. Therefore, there is a need to design ethnically tailored strategies to modify the lifestyles of different populations. Because the factors affecting Asian diabetes patient are not always the same as those in highincome Western countries [54], we encourage the medical professionals in Asian countries to perform more studies that control for variability in ethnicity to reach a better conclusion.

Furthermore, a healthy lifestyle behavior and attitude will be effective for improving the glucose balance, so the promotion of healthy school policies is needed to be wildly implemented in school environment, such as providing healthier meals and achieving physical activity goals in different age students to prevent children to become obese adults [57]. In particular, type 2 diabetes is associated with obesity and plasma glucose level. Treatment should not only normalize hyperglycemia but also focus on lifestyle modification [58].

For the early prevention of type 2 diabetes among children, interdisciplinary teamwork may improve the intervention integrity. For example, school nurses could provide a regular screening and refer high-risk students to general practitioners and dialectologists. Multiple approaches should be used to maintain energy balance in developing children, including nutritional education and physical activity. We strongly recommend the introduction of playful gym classes or interactive programs into daily life to increase energy expenditure. Furthermore, future studies are needed to examine the dose-response intervention, the effect of follow-up duration, and the cost-effectiveness of lifestyle modification programs to prevent diabetes in overweight/obese children.

Some limitations merit attention. First, the included studies had heterogeneity of the interventions of life style modifications. Second, the studies reported in the metaanalysis were conducted in the age range including puberty. Hormonal changes and gender effect at puberty might affect insulin sensitivity and glucose metabolism. Third, majority of the included studies were conducted in Western countries. However, the effect of lifestyle modification shown in this study still could act as a pilot and serve as a reference for future high-quality randomized controlled trials.

\section{Conclusion}

Lifestyle modification can modestly reduce FPG in overweight/obese children. Further large-scale studies with longer follow-up duration are warranted to examine the effects of lifestyle modification on type 2 diabetes mellitus prevention, especially in Asian countries.

\section{Conflicts of Interest}

The authors have no financial conflicts of interest and no industrial support.

\section{Authors' Contributions}

Yi-Der Jiang and Hung-Rong Yen contributed equally to this work.

\section{Acknowledgments}

The authors thank the authors who shared their data with us for this study, with special thanks to Dr. Hsien-Ho Lin from the Graduate Institute of Epidemiology and Preventive Medicine, College of Public Health, National Taiwan University, for providing consultation on manuscript formation and data integrity. Yi-Der Jiang was supported by Grant MOST-104-2314-B-002-155 from the Ministry of Science and Technology, Taiwan. Hung-Rong Yen was supported by China Medical University under the Aim for the Top University Plan of the Ministry of Education and Department of Chinese Medicine and Pharmacy, Ministry of Health and Welfare, Taiwan (MOHW105-CMAP-M-114-000108).

\section{References}

[1] Z. T. Bloomgarden, "Type 2 diabetes in the young: the evolving epidemic," Diabetes Care, vol. 27, no. 4, pp. 998-1010, 2004.

[2] R. Lavizzo-Mourey, "Childhood obesity: what it means for physicians," Journal of the American Medical Association, vol. 298, no. 8, pp. 920-922, 2007.

[3] World Health Organization (WHO), Childhood overweight and obesity: World Health Organization; 2015, Available from: http://www.who.int/dietphysicalactivity/childhood/en/.

[4] World Obesity Federation, World map of obesity in children 2015. Available from: http://www.worldobesity.org/?map= children.

[5] N.-F. Chu and W.-H. Pan, "Prevalence of obesity and its comorbidities among schoolchildren in Taiwan," Asia Pacific Journal of Clinical Nutrition, vol. 16, no. 2, pp. 601-607, 2007.

[6] S. Parmet, C. Lynm, and R. M. Glass, "JAMA patient page. Weight and diabetes," JAMA, vol. 295, no. 11, p. 1330, 2006.

[7] F. R. Kaufman, K. Hirst, B. Linder et al., "Risk factors for type 2 diabetes in a sixth-grade multiracial cohort: the healthy study," Diabetes Care, vol. 32, no. 5, pp. 953-955, 2009.

[8] M. S. Schwartz and A. Chadha, "Type 2 diabetes mellitus in childhood: obesity and insulin resistance," Journal of the American Osteopathic Association, vol. 108, no. 9, pp. 518-524, 2008.

[9] J.-N. Wei, F.-C. Sung, C.-C. Lin, R.-S. Lin, C.-C. Chiang, and L.-M. Chuang, "National surveillance for type 2 diabetes mellitus in Taiwanese children," Journal of the American Medical Association, vol. 290, no. 10, pp. 1345-1350, 2003.

[10] M. I. Constantino, L. Molyneaux, F. Limacher-Gisler et al., "Long-term complications and mortality in young-onset diabetes: Type 2 diabetes is more hazardous and lethal than type 1 diabetes," Diabetes Care, vol. 36, no. 12, pp. 3863-3869, 2013.

[11] R. W. Simpson, J. E. Shaw, and P. Z. Zimmet, "The prevention of type 2 diabetes - lifestyle change or pharmacotherapy? a challenge for the 21st century," Diabetes Research and Clinical Practice, vol. 59, no. 3, pp. 165-180, 2003.

[12] J. W. Anderson, C. W. C. Kendall, and D. J. A. Jenkins, "Importance of weight management in Type 2 diabetes: review with meta-analysis of clinical studies," Journal of the American College of Nutrition, vol. 22, no. 5, pp. 331-339, 2003. 
[13] UK Prospective Diabetes Study (UKPDS) Group, "Intensive blood-glucose control with sulphonylureas or insulin compared with conventional treatment and risk of complications in patients with type 2 diabetes (UKPDS 33)," The Lancet, vol. 352, no. 9131, pp. 837-853, 1998.

[14] M. I. Goran, G. D. C. Ball, and M. L. Cruz, "Cardiovascular endocrinology 2: Obesity and risk of type 2 diabetes and cardiovascular disease in children and adolescents," Journal of Clinical Endocrinology and Metabolism, vol. 88, no. 4, pp. 14171427, 2003.

[15] J. Steinberger and S. R. Daniels, "Obesity, insulin resistance, diabetes, and cardiovascular risk in children: an American heart association scientific statement from the atherosclerosis, hypertension, and obesity in the young committee (council on cardiovascular disease in the young) and the diabetes committee (council on nutrition, physical activity, and metabolism)," Circulation, vol. 107, no. 10, pp. 1448-1453, 2003.

[16] The Diabetes Prevention Program Research Group, "The diabetes prevention program (DPP): description of lifestyle intervention," Diabetes Care, vol. 25, no. 12, pp. 2165-2171, 2002.

[17] S. E. Inzucchi, R. M. Bergenstal, J. B. Buse et al., "Management of hyperglycemia in type 2 diabetes: a patient-centered approach: position statement of the American Diabetes Association (ADA) and the European Association for the Study of Diabetes (EASD)," Diabetes Care, vol. 35, no. 6, pp. 1364-1379, 2012.

[18] J. Lindström, A. Louheranta, M. Mannelin et al., "The Finnish Diabetes Prevention Study (DPS): lifestyle intervention and 3year results on diet and physical activity," Diabetes Care, vol. 26, no. 12, pp. 3230-3236, 2003.

[19] X.-R. Pan, G.-W. Li, Y.-H. Hu et al., "Effects of diet and exercise in preventing NIDDM in people with impaired glucose tolerance: the Da Qing IGT and diabetes study," Diabetes Care, vol. 20, no. 4, pp. 537-544, 1997.

[20] J. Tuomilehto, J. Lindström, J. G. Eriksson et al., "Prevention of type 2 diabetes mellitus by changes in lifestyle among subjects with impaired glucose tolerance," The New England Journal of Medicine, vol. 344, no. 18, pp. 1343-1350, 2001.

[21] A. Baillot, A. J. Romain, K. Boisvert-Vigneault et al., "Effects of lifestyle interventions that include a physical activity component in class II and III obese individuals: A systematic review," PLoS ONE, vol. 10, no. 4, Article ID e0119017, 2015.

[22] J. X. Jiang, X. L. Xia, T. Greiner, G. L. Lian, and U. Rosenqvist, "A two year family based behaviour treatment for obese children," Archives of Disease in Childhood, vol. 90, no. 12, pp. 1235-1238, 2005.

[23] A. S. Kelly, A. M. Metzig, K. D. Rudser et al., "Exenatide as a weight-loss therapy in extreme pediatric obesity: A randomized, controlled pilot study," Obesity, vol. 20, no. 2, pp. 364-370, 2012.

[24] B. Caballero, T. Clay, and SM. Davis, "Pathways, a schoolbased, randomized controlled trial for the prevention of obesity in American Indian schoolchildren," The American Journal of Clinical Nutrition, vol. 78, no. 5, p. 1030, 2003.

[25] T. S. Hannon, G. Rao, and S. A. Arslanian, "Childhood obesity and type 2 diabetes mellitus," Pediatrics, vol. 116, no. 2, pp. 473480, 2005.

[26] U. Ekelund, J. Luan, L. B. Sherar, D. W. Esliger, P. Griew, and A. Cooper, "Moderate to vigorous physical activity and sedentary time and cardiometabolic risk factors in children and adolescents," The Journal of the American Medical Association, vol. 307, no. 7, pp. 704-712, 2012.
[27] D. Moher, A. Liberati, J. Tetzlaff, and D. G. Altman, "Preferred reporting items for systematic reviews and meta-analyses: the PRISMA statement," Annals of Internal Medicine, vol. 151, no. 4, pp. 264-269, 2009.

[28] T. J. Cole, M. C. Bellizzi, K. M. Flegal, and W. H. Dietz, "Establishing a standard definition for child overweight and obesity worldwide: international survey," British Medical Journal, vol. 320, no. 7244, pp. 1240-1243, 2000.

[29] M. Onis, "WHO Child Growth Standards based on length/ height, weight and age," Acta Paediatrica, vol. 95, pp. 76-85, 2006.

[30] W. Chen and M.-H. Chang, "New growth charts for taiwanese children and adolescents based on world health organization standards and health-related physical fitness," Pediatrics and Neonatology, vol. 51, no. 2, pp. 69-79, 2010.

[31] D. Moher, L. Shamseer, and M. Clarke, "Preferred reporting items for systematic review and meta-analysis protocols (PRISMA-P) 2015 statement," Systematic Reviews, vol. 4, no. 1, article 1, 2015.

[32] T. K. Harrell, B. M. Davy, J. L. Stewart, and D. S. King, "Effectiveness of a school-based intervention to increase health knowledge of cardiovascular disease risk factors among rural Mississippi middle school children," Southern Medical Journal, vol. 98, no. 12, pp. 1173-1180, 2005.

[33] N. Singhal, A. Misra, P. Shah, and S. Gulati, "Effects of controlled school-based multi-component model of nutrition and lifestyle interventions on behavior modification, anthropometry and metabolic risk profile of urban Asian Indian adolescents in North India," European Journal of Clinical Nutrition, vol. 64, no. 4, pp. 364-373, 2010.

[34] R. P. Treviño, Z. Yin, A. Hernandez, D. E. Hale, O. A. Garcia, and C. Mobley, "Impact of the Bienestar school-based diabetes mellitus prevention program on fasting capillary glucose levels: A randomized controlled trial," Archives of Pediatrics and Adolescent Medicine, vol. 158, no. 9, pp. 911-917, 2004.

[35] A. D. A. Boudreau, D. S. Kurowski, W. I. Gonzalez, M. A. Dimond, and N. M. Oreskovic, "Latino families, primary care, and childhood obesity: a randomized controlled trial," American Journal of Preventive Medicine, vol. 44, no. 3, pp. S247-S257, 2013.

[36] A. L. Carrel, R. R. Clark, S. E. Peterson, B. A. Nemeth, J. Sullivan, and D. B. Allen, "Improvement of fitness, body composition, and insulin sensitivity in overweight children in a school-based exercise program: a randomized, controlled study," Archives of Pediatrics and Adolescent Medicine, vol. 159, no. 10, pp. 963-968, 2005.

[37] G. D. Foster, B. Linder, T. Baranowski et al., "A school-based intervention for diabetes risk reduction," The New England Journal of Medicine, vol. 363, no. 5, pp. 443-453, 2010.

[38] C. A. Johnston, C. Tyler, B. K. McFarlin et al., "Weight loss in overweight Mexican American children: A randomized, controlled trial," Pediatrics, vol. 120, no. 6, pp. e1450-e1457, 2007.

[39] M. Savoye, S. Caprio, J. Dziura et al., "Reversal of early abnormalities in glucose metabolism in obese youth: Results of an intensive lifestyle randomized controlled trial," Diabetes Care, vol. 37, no. 2, pp. 317-324, 2014.

[40] O. Van Der Baan-Slootweg, M. A. Benninga, A. Beelen et al., "Inpatient treatment of children and adolescents with severe obesity in the Netherlands: A randomized clinical trial," JAMA Pediatrics, vol. 168, no. 9, pp. 807-814, 2014. 
[41] R. C. Vos, J. M. Wit, H. Pijl, and E. C. Houdijk, "Long-term effect of lifestyle intervention on adiposity, metabolic parameters, inflammation and physical fitness in obese children: a randomized controlled trial," Nutrition and Diabetes, vol. 1, no. 10, p. e9, 2011.

[42] Y. Uysal, B. Wolters, C. Knop, and T. Reinehr, "Components of the metabolic syndrome are negative predictors of weight loss in obese children with lifestyle intervention," Clinical Nutrition, vol. 33, no. 4, pp. 620-625, 2014.

[43] J.-J. Wang, W.-C. P. Lau, H.-J. Wang, and J. Ma, "Evaluation of a comprehensive intervention with a behavioural modification strategy for childhood obesity prevention: a nonrandomized cluster controlled trial Health behavior, health promotion and society," BMC Public Health, vol. 15, no. 1, article no. 206, 2015.

[44] J. P. Higgins and D. G. Altman, "Assessing Risk of Bias in Included Studies," Cochrane Handbook for Systematic Reviews of Interventions: Cochrane Book Series, pp. 187-241, 2008.

[45] M. H. L. Borenstein, J. Higgins, and H. Rothstein, Comprehensive Meta-analysis. 2 ed. Englewood, NJ: Biostat; 2005.

[46] J. P. Koplan, C. T. Liverman, V. I. Kraak, and Committee on Prevention of Obesity in Children and Youth, "Preventing childhood obesity: health in the balance: executive summary," Journal of the American Dietetic Association, vol. 105, no. 1, pp. 131-138, 2005.

[47] K. C. Copeland, J. Silverstein, K. R. Moore et al., "Management of newly diagnosed type 2 Diabetes Mellitus (T2DM) in children and adolescents," Pediatrics, vol. 131, no. 2, pp. 364-382, 2013.

[48] C. L. Davis, N. K. Pollock, J. L. Waller et al., "Exercise dose and diabetes risk in overweight and obese children: a randomized controlled trial," JAMA: Journal of the American Medical Association, vol. 308, no. 11, pp. 1103-1112, 2012.

[49] The International Expert Committee, "International Expert Committee report on the role of the $\mathrm{AlC}$ assay in the diagnosis of diabetes," Diabetes Care, vol. 32, no. 7, pp. 1327-1334, 2009.

[50] H.-S. Chen, T.-S. Jap, R.-L. Chen, and H.-D. Lin, "A prospective study of glycemic control during holiday time in type 2 diabetic patients," Diabetes Care, vol. 27, no. 2, pp. 326-330, 2004.

[51] M. Sohmiya, I. Kanazawa, and Y. Kato, "Seasonal changes in body composition and blood HbAlc levels without weight change in male patients with type 2 diabetes treated with insulin," Diabetes Care, vol. 27, no. 5, pp. 1238-1239, 2004.

[52] P.-L. Tsou, Y.-D. Jiang, C.-C. Chang et al., "Sex-related differences between adiponectin and insulin resistance in schoolchildren," Diabetes Care, vol. 27, no. 2, pp. 308-313, 2004.

[53] C.-H. Yu, P.-H. Liu, Y.-H. Van, A. S.-Y. Lien, T.-P. Huang, and H.-R. Yen, "Traditional Chinese medicine for idiopathic precocious puberty: a hospital-based retrospective observational study," Complementary Therapies in Medicine, vol. 22, no. 2, pp. 258-265, 2014.

[54] M. Chiu, P. C. Austin, D. G. Manuel, B. R. Shah, and J. V. $\mathrm{Tu}$, "Deriving ethnic-specific BMI cutoff points for assessing diabetes risk," Diabetes Care, vol. 34, no. 8, pp. 1741-1748, 2011.

[55] A. S.-Y. Lien, Y.-D. Jiang, C.-H. Mou, M.-F. Sun, B.-S. Gau, and H.-R. Yen, "Integrative traditional Chinese medicine therapy reduces the risk of diabetic ketoacidosis in patients with type 1 diabetes mellitus," Journal of Ethnopharmacology, vol. 191, pp. 324-330, 2016.

[56] A. L. Lee, B. C. Chen, C. H. Mou, M. Sun, and H. Yen, "Association of traditional chinese medicine therapy and the risk of vascular complications in patients with type II diabetes mellitus a nationwide, retrospective, taiwanese-registry, cohort study," Medicine, vol. 95, no. 3, pp. 1-7, 2016.

[57] M. Story, M. S. Nanney, and M. B. Schwartz, "Schools and obesity prevention: creating school environments and policies to promote healthy eating and physical activity," The Milbank Quarterly, vol. 87, no. 1, pp. 71-100, 2009.

[58] K. Dileepan and M. Max Feldt, "Type 2 diabetes mellitus in children and adolescents," Pediatrics in Review, vol. 34, no. 12, pp. 541-548, 2013. 


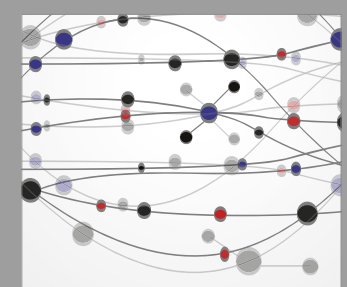

The Scientific World Journal
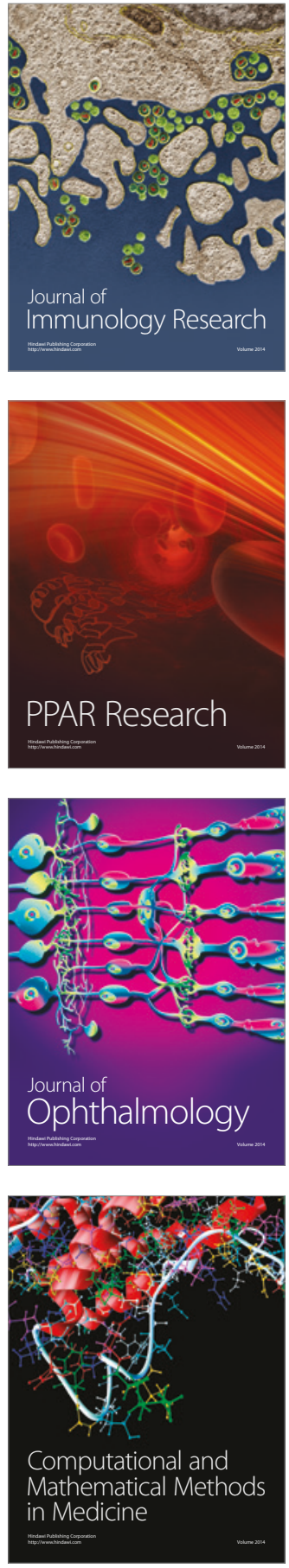

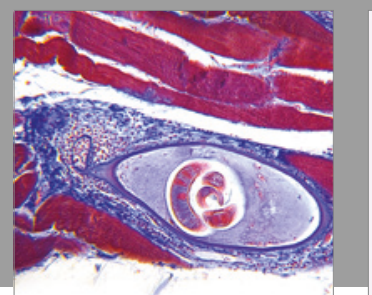

Gastroenterology Research and Practice
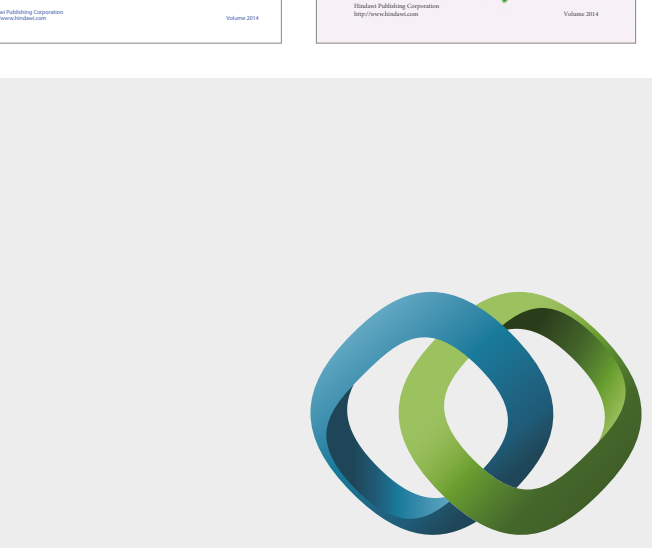

\section{Hindawi}

Submit your manuscripts at

https://www.hindawi.com
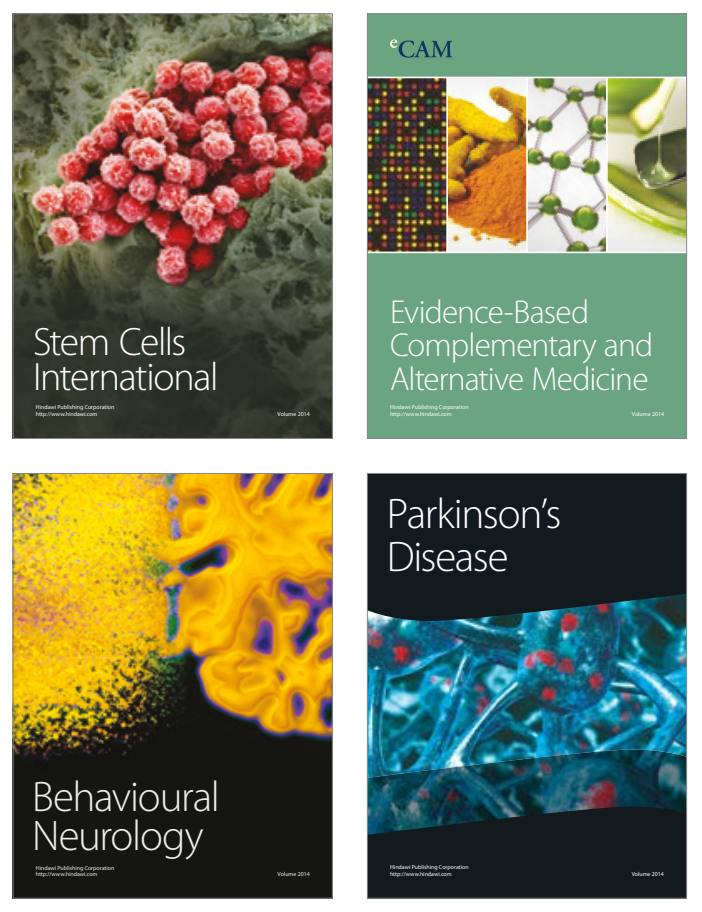
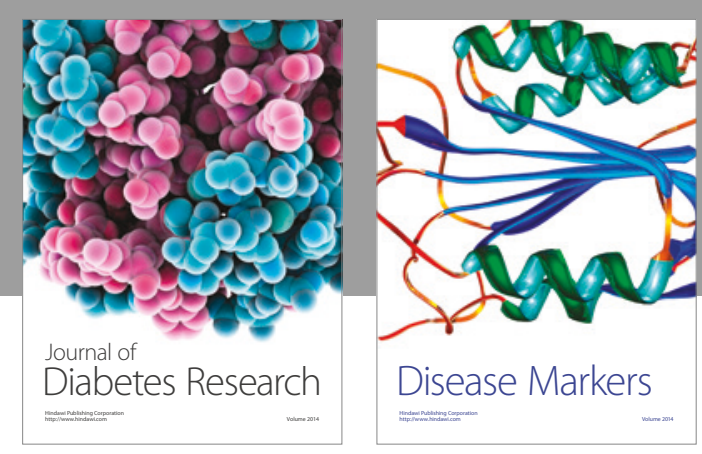

Disease Markers
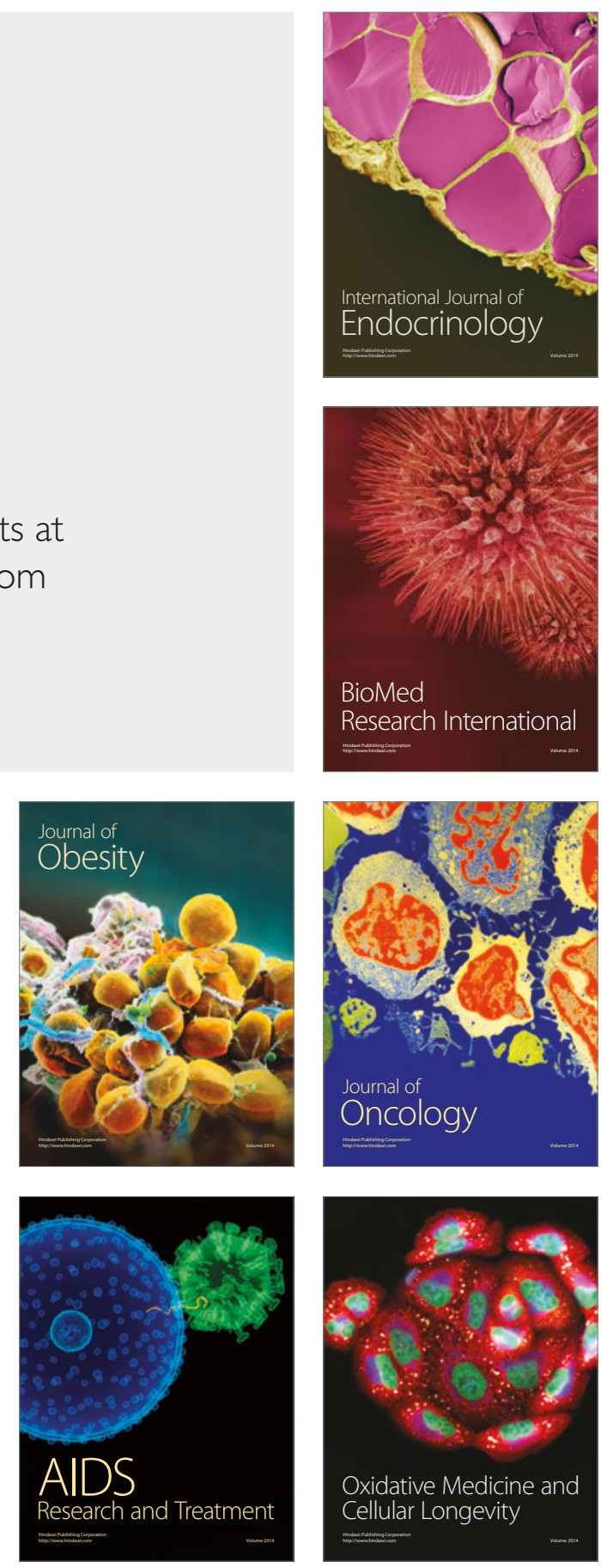